\title{
Design and Implementation of a Dual Master Computer Based Intelligent Tutoring System
}

\author{
Ziming Du \\ \{ duzimingwh@163.com\} \\ College of Light Industry, Wuhan polytechnic Wuhan 430074, China
}

\begin{abstract}
In this paper, the double subject teaching model, teachers play the leading role and can fully reflect student's cognitive main role, more in line with the current situation of China's education development; historical inheritance of study of student model, allowing the system to an accurate assessment of students, the realization of the optimal teaching effect. In addition, this paper presents the concept tree to represent knowledge, which is not only convenient to check the redundancy of knowledge, but also is suitable for students to learn the automatic navigation, it is worth promoting. In the part of design, this paper combines domain knowledge representation method, is given a oriented cognitive object of hyper text knowledge representation method, put forward the rules of teaching strategy reasoning machine based on weighted; according to cognitive learning theory, a by successive approximation method of historical inheritance of study cognitive student model, which design a learning process automatic navigation and hypertext navigation teaching model based on the student model and knowledge presentation. It also discusses the intelligent system to explain the mechanism and man-machine interface design. In the part of realization, the realization of writing environment and man-machine interface, discusses the organization and management of knowledge, gives the reasoning of concrete realization of the code and try to give a intelligent tutoring system model of the initial implementation in the last part of this paper, the prospect of the development of the system are discussed.
\end{abstract}

Keywords: Expert system; intelligent teaching system; double main teaching model; concept tree

\section{Introduction}

Over the years, many excellent teachers in difficult to grasp programming technology and cannot be valuable teaching should be reflected in the design of courseware; and programming highly skilled computer professionals often lack experience in teaching practice, it is difficult to in the program design reflected education theory and method. Therefore introduced most of the courseware belongs to traditional teaching courseware, it for different students, the effect is stereotyped, unable to realize individualized teaching, even intelligent courseware and its intelligent effect is not particularly high, most of the low efficiency, and difficult to make teaching design and program design with the tacit understanding. I think, as a computer educator [1], should have the advantage of these two aspects, this is my design of the original intention of the system. And more important is that due to the different application fields of intelligent teaching system, Professor of the content and object of different. Therefore, 
according to the specific teaching environment by specific intelligent algorithm or model to design a adapt to student's intelligent teaching system becomes more necessary. According to the teaching experience, I put the students into two groups: a class is a new (did not study computer courses), one is old school computer curriculum). For these two types of students, my teaching method is: the former requires controlled learning, which can choose their own learning, implementation of the "teaching" and "learning" the double main teaching mode, the system self breaking can be always around this model and design. The traditional teaching system is to put all the teaching information, in the programming mode is preset in the courseware, the student studies, the teaching letter from, is according to the preset teaching process provides to the student. That is, no matter how the students start, the face of the teaching process will be completely consistent, which obviously is not conducive to the students to play the subjective initiative. Moreover, once the production of such courseware is complete, any larger teaching improvements may lead to the whole software system to re compile, to maintain the work of great inconvenience.

\section{The Teaching Mode and System Characteristics of ITS}

The popular teaching mode has three types: traditional take the teacher as the center of teaching mode; based on the constructivism with the students as the center of teaching mode, can play to the teacher's leading role and fully reflect student's cognitive function "double master" teaching mode. The traditional teaching mode based on the "teacher" is based on the theory of "stimulus response" learning and the teaching theory. In this kind of teaching mode, the teacher is the teaching activity center, is the teaching activity dominant, is the knowledge teaching person, the infusion person; but the student is the object of infusion, is the passive recipient of the outside stimulation. This kind of teaching mode due to the neglect of the students in the learning process of internal psychological changes, without considering they as a subjective role in learning and therefore cannot fully mobilize the enthusiasm of learners, is not conducive to play them as a cognitive subject enthusiasm, initiative. Early teaching system using this teaching mode, in the teaching process, only the students as passive recipients of teaching content, the system does not consider they make various reactions, all the content is according to the teaching sequence to be specified in advance, and not according to the needs of different students and different teaching stages teaching adjustment symplectic for, also cannot effectively excitation students' interest in learning, affects the effectiveness of teaching. Cognitivism learning theory overcomes the disadvantages of behaviorism learning theory and diagnosis of learning is the learners according to their own attitudes, needs, interests, hobbies, etc [2]., using their existing cognitive structures, to the external stimulation provided information, make active choice process of information. It began to emphasize the internal psychological changes of learners of the learning process, emphasize students' cognitive structure and cognitive strategies in the learning process in the role that teachers in the teaching process should be selected to establish contact with the students' cognitive structure of the original teaching materials, to stimulate students to apply new knowledge into the existing knowledge system. At present most intelligent tutoring systems are based on the cognitive theory to guide the teaching process. Because it can fully take into account the individual characteristics of learners, according to the learner's learning level, learning ability, cognitive structure, and so on to determine the content and form of teaching. 


\subsection{The Basic Structure of ITS}

A complete expert system is usually composed of six parts: knowledge base, knowledge base, inference engine, knowledge acquisition mechanism, explanation mechanism and manmachine interface. Knowledge base, context and inference machine which is is currently the main content of the most expert systems, knowledge acquisition mechanism, explanation mechanism and man-machine interface is all expert systems are expected to have three modules, but they did not have been realized. Simple expert systems do not necessarily have these three modules. ITS system mainly in knowledge representation, reasoning methods and natural language understanding of the use of three aspects of artificial intelligence theory. Knowledge representation is used to establish its curriculum knowledge base; reasoning strategy to answer their questions, assessment of a student's work, which is mainly on the knowledge in the knowledge base reasoning, draw the conclusion; natural language understanding makes teaching system allowing students to use natural language dialogue with the computer. Moreover, the computer also decided to when and in what ways students to provide the necessary feedback information. Among them, knowledge representation and reasoning is the core of the development of ITS [3].
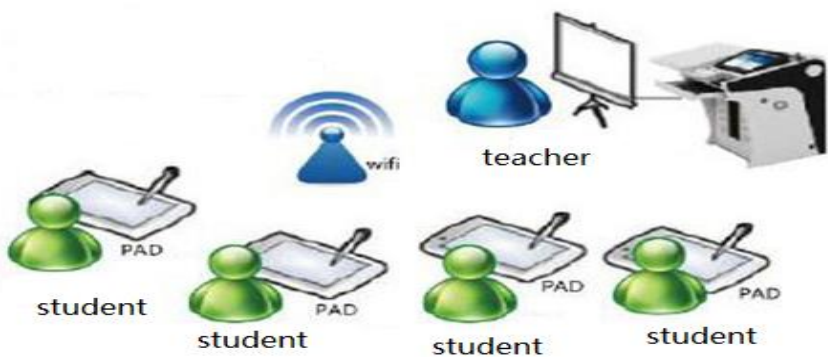

Fig. 1. Schematic diagram of intelligent teaching system

\subsection{ITS Function Introduction}

ITS based on cognitive theory, the application of artificial intelligence technology in teaching system. ITS will be separated from the teaching content and teaching strategies, according to the cognitive model of student's letter J, through the search and reasoning of intelligent systems, dynamic content and strategies suitable for individual teaching; based on the intelligent diagnosis system to determine the level of student learning, students analyze the cause of the error, and propose changes, and further learning content suggestions to students; through the error statistics for all students, the intelligent diagnosis mechanism will give teachers the teaching focus, focus, methods, test questions and suggestions; provide for teachers teaching content, friendly test content maintenance interface, no need to change the teaching strategy to adjust the structure of the software; through intelligent the students' cognitive model, teaching content, analysis the test results, the evaluation of teachers' teaching performance in reference to the teaching supervision personnel. It can be said that an ideal, perfect ITS system is an independent, excellent teacher". With the existing scientific and technical level, the ITS system with the above functions can not be realized in a short period of time. It is generally believed that a teaching system with one or more of the following features can be called a ITS system [4].

(L) Can automatically generate a variety of problems and exercises; 
(2) According to the students' learning level and learning situation, the content and the progress of learning and learning;

(3) Automatically solve the problem on the basis of understanding the teaching content;

(4) With the ability to generate and understand natural language, in order to achieve a more liberal teaching and answering system, to improve the initiative of human-computer interaction;

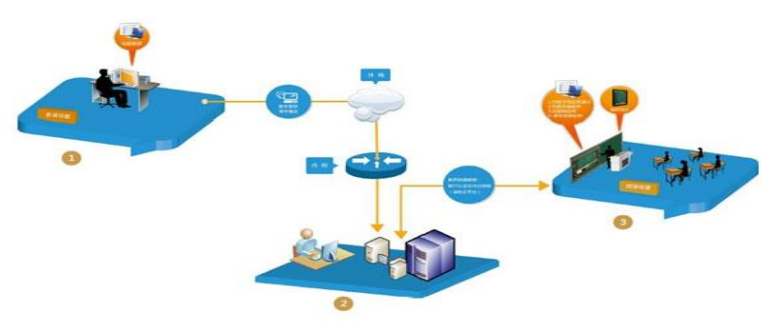

Fig. 2. Teaching system based on artificial intelligence technology

\subsection{Intelligent Writing Environment}

In order to shorten the development cycle of the intelligent teaching system and improve its efficiency, we can also choose a variety of software tools. Most of the software tools, can help the programmer to complete the development of a part or a certain type of work, such as text editor, graphics editor, voice editor, etc.. However, due to the different tool interface standards are inconsistent, and often with the closure and protection, so open software tools are increasingly attracted the attention of developers. In addition, at the same time, the choice of writing language, to consider it and the interface of the software tool, and can not be alone to choose. Oriented object software system structure is in accordance with the problems in the field of model is built up, rather than based on decomposing system to complete the function, so when the functional requirements of the system change does not cause changes of the whole software structure, often only need some local modifications. Therefore, it is more stable to construct the software system with the object as the center.

\section{Knowledge Processing of ITS and DCB}

Knowledge representation method, not only can reasonably and effectively to organize domain knowledge together, for the system to provide information, supply system of query, also can help students to establish a clear conception of the relationship, it is learner should master, also is a kind of cognitive psychology. The advantages and disadvantages of knowledge representation method is very important to the teaching system, good knowledge representation, is advantageous to the curriculum content retrieval, helps the student to establish the good knowledge structure, enhances the problem solving ability. Therefore, the method of knowledge representation is the key to the ITS development system. Frame representation provides a convenient mechanism knowledge base builder domain knowledge description. The main advantage of this method is that it can reflect the way of thinking of experts, which provides a strict structure, and provides convenience for the establishment of efficient inference algorithms. Although the framework has many advantages, but it also has problems, it can not fully meet the above requirements. In order to meet these requirements, 
people have put forward a variety of representation methods. These methods can be basically divided into two categories: description representation and process representation. Explains the general process of representing knowledge as a set of facts and a set of facts that are attached to a group. Process representation method is used to represent the process of using it. The method has many advantages, such as the fact that each fact is stored only once, and no matter the fact that the various methods are different; the new facts are easy to add the system, and do not change other facts or local process. Process representation also has many advantages, such as easy to express how to deal with the problem of knowledge, ease of expression is not suitable to express the knowledge in a way that is easy to express the heuristic knowledge [5].

\section{Conclusion}

Application of expert system is mainly studied in this thesis in the intelligent tutoring system, from the theoretical basis and teaching pattern of intelligent teaching system of build a pair of main intelligent teaching system for computer basic course, the design and implementation of the the system, and provides a collaborative environment for other courses. After nearly a year of research and development, basically be able to put into use. But the expert system development process itself is a cyclic process. Compared with other computer systems and its development cycle may be longer, and it relates to the relevant content than the other computer is also more complex, from the knowledge representation, knowledge acquisition, inference mechanism, solution release mechanism, student model, teacher model, to human-computer interface design each part is a subject. Therefore, this system is only in the knowledge representation, reasoning mechanism, student model, teacher model these aspects have made a more detailed study, other aspects of the need to change the import.

\section{References}

[1] Ting, Fu Gao Lei. Intelligent computer assisted instruction system in the construction of new teaching mode. Journal of China Coal Economic College. No. 03. (2015)

[2] Pu Ying, Liu Xiao Fang, Jiang Xiaohua, Luo Ying Li, Zhang Jianhua. (II) -- intelligent training system research on the key technology of electrical workers intelligent training system of knowledge said. Modern electric power. No. 02. (2015)

[3] Lu Yi, Feng Demin. ICAI system simulation to achieve. Journal of Shaanxi Normal University (NATURAL SCIENCE EDITION). No. 02. (2011)

[4] Qi Hui, Guo Xin. Design and implementation of intelligent computer and auxiliary teaching system. Journal of Henan University (NATURAL SCIENCE EDITION). No. 03. (2012)

[5] Rong Yang, Wang Huifang, Le Yujun. Courseware development platform in teaching and learning strategy for the design of studies. Tianjin Normal University Journal (NATURAL SCIENCE EDITION). No. 04. (2013) 